\title{
Protein expression diversity amongst serovars of Salmonella enterica
}

\author{
Correspondence \\ H. N. Shah \\ Haroun.Shah@hpa.org.uk
}

Received 30 May 2007

Revised 6 August 2007

Accepted 20 August 2007
V. Encheva, ${ }^{1}$ R. Wait, ${ }^{2}$ S. Begum, ${ }^{2}$ S. E. Gharbia ${ }^{3}$ and H. N. Shah ${ }^{1}$

\author{
${ }^{1}$ Molecular Identification Services Unit, Centre for Infections, Health Protection Agency, \\ 61 Colindale Avenue, London NW9 5EQ, UK \\ ${ }^{2}$ Kennedy Institute of Rheumatology Division, Faculty of Medicine, Imperial College, ARC Building, \\ 1 Aspenlea Road, London W6 8LH, UK \\ ${ }^{3}$ Applied and Functional Genomics Unit, Centre for Infections, Health Protection Agency, \\ 61 Colindale Avenue, London NW9 5EQ, UK
}

\begin{abstract}
Salmonella enterica is one of the most extensively studied bacterial species in terms of physiology, genetics, cell culture and development. As a very diverse group, the serovars of $S$. enterica display a spectrum of host specificities ranging from a broad host range to strictly hostadapted variants. This study utilized a classic proteomic approach combining 2D gel electrophoresis and mass spectrometry for the comparative analysis of the proteomes of serovars Typhimurium, Enteritidis, Choleraesuis, Pullorum and Dublin. The comparative analysis revealed species-specific protein factors with no significant change in expression amongst all isolates, as well as proteins with fluctuating expression levels between serovars and strains. Examples include an isoform of SodA specific for serovar Typhimurium, the third isoform of the lysine arginine ornithine (LAO)-binding amino acid transporter specific for serovar Pullorum, and the enzyme GabD found to be unique to serovar Choleraesuis. Overall the study demonstrated the importance of using multiple isolates when characterizing the expression patterns of bacteria in order to account for the intrinsic diversity of a bacterial population and revealed several factors with potential roles in host adaptation and pathogenicity of the serovars of $S$. enterica.
\end{abstract}

\section{INTRODUCTION}

Salmonellae are significant not only as an ongoing threat to worldwide public health, but also as a model system for the study of fundamental mechanisms of bacterial pathogenesis. Salmonella enterica subspecies enterica is routinely divided into serovars on the basis of expression of three surface antigens (Ags), the somatic $\mathrm{O} \mathrm{Ag}$, the flagellar $\mathrm{H} 1$ and $\mathrm{H} 2 \mathrm{Ags}$, and the capsular $\mathrm{Vi} \mathrm{Ag}$, according to the Kauffmann-White scheme (Popoff et al., 2003). Although the serovars are very closely related, they have different host ranges and cause different disease symptoms (Baümler, 1997). Serovars Typhimurium and Enteritidis are generalists, since they infect a wide range of animals (including wild rodents, poultry, pigs and cattle) and cause gastroenteritis in humans, systemic infection in mice and asymptomatic chronic infection in chickens. Serovars Choleraesuis and Dublin are host-adapted, infecting only a few species. Serovar Choleraesuis primarily infects swine, while serovar Dublin infects cattle, although these serovars can cause disease in other animals (Baümler et al., 1998).

Abbreviations: GE, gel electrophoresis; LC, liquid chromatography.

A supplementary table of the density values for all protein spots measured in this study is available with the online version of this paper.
Other serovars are host-specific, infecting only one animal host, e.g. serovar Pullorum infects only poultry and serovar Typhi infects only humans. Furthermore, the serovars show different levels of virulence, e.g. the host generalists such as Enteritidis and Typhimurium tend to colonize young animals, which suggests that they struggle to adapt to a fully mature immune system. Host-adapted serovars, on the other hand, tend to cause disease with equal frequency in all age groups and are more virulent, which is illustrated by the higher mortality rates they exhibit (Baümler et al., 1998). Initial DNA hybridization studies have shown that the serovars of S. enterica share $>90 \%$ of their DNA content (Crosa et al., 1973). Comparison of their genomes has revealed that, despite their similarity, each serovar has many insertions and deletions relative to other serovars, which range in size from 1 to $50 \mathrm{~kb}$ and are distributed throughout the chromosome (Edwards et al., 2002). In total, each serovar has approximately 500-600 kb of unique DNA, which represents $10-12 \%$ of their genomes (Edwards et al., 2002). However, these differences observed at the DNA level have not been related to protein expression.

The protein expression patterns of S. enterica serovar Typhimurium have been extensively studied, and an 
annotated reference map of the cytosolic and cell envelope proteins of this serovar has been published (Encheva et al., 2005; Qi et al., 1996; O'Connor et al., 1997). A reference map of the cytosolic proteins expressed by serovar Enteritidis has also been created (Park et al., 2003), but the expression patterns of the two serovars have not been compared. Furthermore, all of the above investigations have been performed on a limited number of strains and the variation in protein expression between a large number of isolates has never been assessed.

The objective of the present investigation was to consider the level of variation in the protein expression patterns of closely related Salmonella serovars, in order to search for protein factors with levels of expression or post-translational modifications characteristic for each serovar. In an earlier study, by using comparative 2D gel electrophoresis (GE) analyses of the two serovars Typhimurium and Pullorum, we demonstrated the presence of several differentially expressed proteins resulting from otherwise identical genetic material (Encheva et al., 2005). In the current study we have broadened this approach, extending it to include different serovars of Salmonella with very different host preferences and pathogenic potentials. The focus of the study was on serovars Typhimurium, Enteritidis, Choleraesuis, Pullorum and Dublin, which are associated with the majority of infections of mammalian and avian hosts (Baümler et al., 1998).

\section{METHODS}

Bacterial strains and growth conditions. A total of 12 strains of $S$. enterica representing five different serovars, including reference strains and clinical isolates, were used in this study. Five type strains, representing serovar Typhimurium (strains 74 and 12023), Enteritidis (strain 12694), Pullorum (strain 10704) and Dublin (strain 12709), were obtained from the National Collection of Type Cultures (Health Protection Agency, London, UK). Three additional strains, representing serovar Choleraesuis (strains B5 and B7) and serovar Pullorum (strain B52), were obtained from the Salmonella Reference Collection at the University of Calgary, Canada. The clinical isolates were supplied by the Department of Risk Research of the Veterinary Laboratories Agency (VLA; Weybridge, UK); they included two strains of serovar Typhimurium (strains 204 and 227), one strain of serovar Dublin (strain 193) and one strain of serovar Enteritidis (strain 97). The bacterial cells were grown on Colombia Blood Agar (CBA; Oxoid) for $18 \mathrm{~h}$ at $37^{\circ} \mathrm{C}$. Extracts were prepared from the second inoculum of each strain and each biological replicate was prepared independently on a different day under identical growth conditions.

Sample preparation. All chemicals were obtained from Sigma unless otherwise specified.

Cell lysates were obtained by harvesting the growth of three plates of S. enterica and resuspending the cells in $20 \mathrm{mM}$ Tris ( $\mathrm{pH} 7.5$ ). The cells were washed and collected by centrifugation at $5100 \mathrm{~g}$ for $10 \mathrm{~min}$. The resulting cell pellet was resuspended in $100 \mu \mathrm{l}$ lysis solution containing $0.3 \%(\mathrm{w} / \mathrm{v})$ SDS, $200 \mathrm{mM}$ DTT and $50 \mathrm{mM}$ Tris

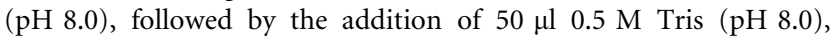
$50 \mathrm{mM} \mathrm{MgCl}_{2}, 1 \mathrm{mg}$ DNase I ml ${ }^{-1}$ and $0.25 \mathrm{mg}$ RNase A ml${ }^{-1}$. The samples were vortexed with $0.3 \mathrm{~g}$ glass beads $(<105 \mu \mathrm{m}$ diameter $)$ and homogenized five times for $1 \mathrm{~min}$ using a Mickle Cell Disintegrator (Mickle Laboratory Engineering) with $1 \mathrm{~min}$ cooling time on ice in between runs. The unbroken cells and cell debris were removed by centrifugation at $21000 \mathrm{~g}$ for $30 \mathrm{~min}$ at $4{ }^{\circ} \mathrm{C}$, and the resulting supernatant was used for further analysis.

Rehydration and IEF. Protein samples containing a total of $150 \mu \mathrm{g}$ protein were mixed with rehydration buffer [7 $\mathrm{M}$ urea, $2 \mathrm{M}$ thiourea, $2 \%$ (w/v) CHAPS, $50 \mathrm{mM}$ DTT, $0.5 \%$ (v/v) IPG buffer pH 3-10 NL (Amersham BioSciences)]. The IPG strips were rehydrated by the ingel rehydration method (Rabilloud et al., 1994) using $18 \mathrm{~cm}$ IPG strips ( $\mathrm{pH} 3-10 \mathrm{NL}$, Amersham BioSciences). The samples were left to rehydrate for $18 \mathrm{~h}$ at $20{ }^{\circ} \mathrm{C}$. IEF was performed using an Investigator 5000 (Genomic Solutions) power supply for $24 \mathrm{~h}$, for a total of $85000 \mathrm{~V} \mathrm{~h}$ at a maximum voltage of $5000 \mathrm{~V}$ and a maximum current of $110 \mu \mathrm{A}$.

Equilibration of the IPG strips and second-dimension separation. The focused IPG strips were equilibrated in Tris/acetate equilibration buffer (Genomic Solutions) containing $0.5 \%(\mathrm{w} / \mathrm{v})$ SDS, $30 \%(\mathrm{v} / \mathrm{v})$ glycerol and $6 \mathrm{M}$ urea. The equilibration was performed in two consecutive steps of $30 \mathrm{~min}$ each. The first step was conducted in the presence of $50 \mathrm{mM}$ DTT, and the second in the presence of $135 \mathrm{mM}$ iodoacetamide.

The second-dimension separation was performed on $10 \%$ Duracryl gels (Proteomic Solutions). The gels were run for $5 \mathrm{~h}$, at a maximum voltage of $500 \mathrm{~V}$ and maximum power of $20000 \mathrm{~mW}$ per gel using the Investigator 5000 power supply (Genomic Solutions).

The gels were post-stained with fluorescent dye SYPRO Ruby (Molecular Probes) for $18 \mathrm{~h}$ with constant shaking and destained for $2 \mathrm{~h}$ in $10 \%(\mathrm{v} / \mathrm{v})$ methanol. The resulting 2D GE profiles were visualized using the Typhoon Scanner (Amersham Biosciences). To excise the spots the gels were double stained with Colloidal $G$ and destained in $25 \%(\mathrm{v} / \mathrm{v})$ methanol.

Analysis of the 2D GE profiles. For the comparative analysis duplicate experimental samples from two different batches of each isolate were used (two technical replicates and two biological replicates for each isolate). The density/volume of the SYPRO Ruby-stained protein spots were determined using the Proteome Weaver software package (Definiens). For a protein to be reported as differentially expressed, the density values measured in Proteome Weaver were further subjected to Student's $t$-test, and only changes with $P<0.01$ were considered significant. The density values for all protein spots measured in this study are given in Supplementary Table S1, available with the online version of this paper.

In-gel trypsin digestion and peptide analysis using liquid chromatography-tandem mass spectrometry (LC MS/MS). Ingel digestion with trypsin was performed according to published methods (Jeno et al., 1995; Wilm et al., 1996; Shevchenko et al., 1996) modified for use with a robotic digestion system (Investigator ProGest, Genomic Solutions) (Wait et al., 2001). Colloidal G was removed by sequential washing with $50 \mathrm{mM}$ ammonium hydrogen carbonate buffer and acetonitrile. Cysteine residues were reduced with DTT and derivatized by treatment with iodoacetamide. After further washing with ammonium hydrogen carbonate buffer, the gel pieces were again dehydrated with acetonitrile and dried at $60{ }^{\circ} \mathrm{C}$, prior to the addition of modified trypsin (Promega; $10 \mu \mathrm{l}$ of a $6.5 \mathrm{ng} \mu \mathrm{l}^{-1}$ solution in $25 \mathrm{mM}$ ammonium hydrogen carbonate). Digestion proceeded for $8 \mathrm{~h}$ at $37^{\circ} \mathrm{C}$, and the products were recovered by sequential extractions with $25 \mathrm{mM}$ ammonium hydrogen carbonate, $5 \%(\mathrm{v} / \mathrm{v})$ formic acid, and acetonitrile. The pooled extracts were lyophilized and redissolved in $0.1 \%(\mathrm{v} / \mathrm{v})$ formic acid for mass spectrometry. 
Tandem electrospray mass spectra were recorded using a Q-TOF hybrid quadrupole/orthogonal acceleration time of flight spectrometer (Micromass) interfaced to a Micromass CapLC capillary chromatograph. Samples were dissolved in $0.1 \%(\mathrm{v} / \mathrm{v})$ formic acid, and $6 \mu \mathrm{l}$ was injected onto a Pepmap C18 trap column (300 $\mu \mathrm{m} \times 0.5 \mathrm{~cm}$; LC Packings), and washed for 3 min with $0.1 \%$

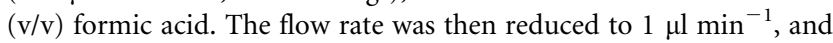
the peptides were eluted into the mass spectrometer with an acetonitrile $/ 0.1 \%(\mathrm{v} / \mathrm{v})$ formic acid gradient [5-70\% (v/v) acetonitrile over $20 \mathrm{~min}]$.

The capillary voltage was set to $3500 \mathrm{~V}$, and data-dependent MS/MS acquisitions were performed on precursors with charge states of 2, 3 or 4 over a survey mass range of 540-1200 Da. Known trypsin autolysis products and keratin-derived precursor ions were automatically excluded. The collision voltage was varied between 18 and $45 \mathrm{~V}$ depending on the charge and mass of the precursor. Product ion spectra were charge state de-encrypted and de-isotoped with a maximum entropy algorithm (MaxEnt 3, Micromass). Proteins were identified by correlation of uninterpreted tandem mass spectra to entries in SWISS-PROT/TREMBL (Wait et al., 2002) using the ProteinLynx Global Server (Version 1.1, Micromass) and NCBInr using MASCOT. One missed cleavage per peptide was allowed, and an initial mass tolerance of 50 p.p.m. was used in all searches. Cysteines were assumed to be carbamidomethylated, but other potential modifications were not considered in the first-pass search.

All matching spectra were reviewed manually, but in cases where the score reported by ProteinLynx global server was less than 100, additional searches were performed against the NCBI nr database using MASCOT, which utilizes a robust probalistic scoring algorithm (Perkins et al., 1999). Identifications based on a single matching peptide were verified manually using the MassLynx program Pepseq (Waters). A list of all proteins identified by LC/MS/MS in this study is given in Table 1.

\section{RESULTS AND DISCUSSION}

\section{Serovar Typhimurium}

When comparing the profile of serovar Typhimurium to the other serovars, it became apparent that two mobility forms of the $\mathrm{Mn}^{2+}$-binding superoxide dismutase (SodA) were present. The two forms have identical molecular mass $(21000 \mathrm{Da})$ but differ in their pI. All isolates of serovar Typhimurium overexpressed isoform II while the other serovars overexpressed isoform I (Fig. 1). The existence of two isoforms of SodA has not been reported previously, and it is not clear if they are the result of amino acid substitutions or post-translational modifications. When comparing the sequences of SodA of serovars Typhimurium and Choleraesuis, slight variation in the amino acid sequences was observed. This contributes to a minor difference in the predicted molecular mass of SodA, but does not contribute to a change in the predicted pI. Therefore the presence of two different isoforms of SodA with different $\mathrm{pI}$ values does not appear to be a result of sequence variation at the protein level.

It has been reported previously that SodA protects Salmonella from an early oxygen-dependent killing in J774 macrophages, but sodA mutants have been shown to be only mildly attenuated in mice (Tsolis et al., 1995).
Another superoxide dismutase was also detected in the 2D GE profile of all Salmonella strains, an $\mathrm{Fe}^{2+}$-binding SodC (data not shown). It has been reported that mutants lacking both $\operatorname{sod} C$ and $\operatorname{sod} A$ are attenuated in mice (van der Straaten et al., 2004). It can be surmised that SodA is important for survival in host macrophages but when mutated, its action is probably substituted by the presence of another enzyme with Sod activity. The two forms of the enzyme observed here need to be investigated further in order to ascertain if they have different activities, and whether this is relevant to the host-specificity of the serovars.

The high degree of variation between isolates of serovar Typhimurium resulted in the detection of only one protein isoform that appeared to be specific to this serovar (SodA isoform II). Several differences in expression were observed between clinical isolates and reference strains, where the reference strains showed elevated expression levels of two transport proteins, periplasmic oligopeptide-binding protein precursor (OppA) and periplasmic $A B C$ superfamily dipeptide transporter (DppA) (Fig. 2). Similarly, the clinical strains showed elevated expression levels of two unidentified proteins. A putative aldehyde dehydrogenase was detected in the expression maps of all but two isolates, 227 and 12023 (Fig. 2). It appears that, depending on the origin of the strains, different sets of proteins exhibit elevated expression levels. The high expression levels of transport proteins in reference strains maintained in the laboratory and the characteristic overexpression of the two unidentified proteins in the clinical isolates may be a response to the different nutritional background. However, some differences in the expression patterns, as in the case of the putative aldehyde dehydrogense, are unrelated to sero-specificity or origin of the strains. Post-translational modification or amino acid substitution may have altered the migration position of this protein in the 2D GE patterns of strains 12023 and 227.

\section{Serovar Enteritidis}

When comparing the expression patterns of serovars Enteritidis and Typhimurium two isoforms of another transport protein, D-galactose-binding protein precursor (MglB) were observed. Similarly to SodA, the two isoforms had an identical molecular mass but a slightly different $\mathrm{pI}$ (Fig. 3). The isolates of serovar Typhimurium overexpressed isoform I while the isolates of serovar Enteritidis overexpressed the second isoform.

$\mathrm{MglB}$ is a part of a periplasmic transporter system which is involved in the transport of galactose and glucose (Death \& Ferenci, 1993). Despite the presence of the phosphotransferase system (PTS), whose role in transport and phosphorylation of glucose across the cytoplasmic membrane is well established, the MglB-dependent system has been shown to be involved predominantly in glucose transport under glucose-limiting conditions (Death \& Ferenci, 1993). Under such conditions, which S. enterica 
Table 1. List of all identified proteins, their corresponding SWISS-PROT identifiers, unique gene names, gene identifiers, sequences of the peptides detected by LC MS-MS and references to the appropriate figures

\begin{tabular}{|c|c|c|c|c|}
\hline Protein name & SWISS-PROT identifier & Gene name/ORF & Peptide sequence obtained & Figure reference \\
\hline $\begin{array}{l}\text { ABC superfamily dipeptide } \\
\text { transport protein }\end{array}$ & Q8Z299 & $d p p A / S T M 3630$ & $\begin{array}{l}\text { (K)AVYQG AGVAA K(N) } \\
\text { (K)IVTYE WGEYL K(R) } \\
\text { (K)ELNAD DVVFS FDR(Q) } \\
\text { (K)AFDGY WGTKP QIDR(L) } \\
\text { (K)WCYKP FEDLI QPAR(A) } \\
\text { (K)TGTTE VIPGL AEKWD ISEDG(T) } \\
\text { (K)GFTID LWAMP VQRPY NPNAR(R) } \\
\text { (K)AGTPE KVDLN PVGTG PFQLV } \\
\text { QYQK(D) }\end{array}$ & Fig. 2(a) \\
\hline $\begin{array}{l}\text { ADP-L-glycero-D-manno- } \\
\text { heptose-6-epimerase }\end{array}$ & HLDD_SALTY & $r f a D / S T M 3710$ & $\begin{array}{l}\text { (R)TSDFI ESR(E) } \\
\text { (R)YQAFT QADLT NLR(N) }\end{array}$ & Fig. 3(a) \\
\hline Alcohol dehydrogenase & Q8XG17 & adhP/STM1567 & $\begin{array}{l}\text { (K)AAFNS AVDAV R(A) } \\
\text { (K)TGGAH AAVVT AVAK(A) } \\
\text { (K)VIAID VNDGQ LK(L) } \\
\text { (R)QDLTE AFQFA AEGK(V) } \\
\text { (K)AAVVT QDHQV DVTEK(T) }\end{array}$ & Data not shown \\
\hline Aldehyde dehydrogenase B & Q8ZL72 & aldB/STM3680 & $\begin{array}{l}\text { (K)EGYYL EPTIL FGK(N) } \\
\text { (R)ETSAA DVPLA IFHFR(Y) } \\
\text { (R)VFQEE IFGPV LAVTT FK(T) }\end{array}$ & Data not shown \\
\hline $\begin{array}{l}\text { D-Galactose-binding periplasmic } \\
\text { protein precursor }\end{array}$ & DGAL_SALTY & $m g l B / S T M 2190$ & $\begin{array}{l}\text { (K)GEPGH PDAEA R(T) } \\
\text { (K)ESGVI QGDLI AK(H) } \\
\text { (R)GQNVP VVFFN K(E) } \\
\text { (K)QNDQI DVLLA K(G) } \\
\text { (K)ALAIN LVDPA AAGTV IEK(H) } \\
\text { (R)VPYVG VDKDN LSEFT QK(E) }\end{array}$ & Fig. 3(a) spot 1. \\
\hline $\begin{array}{l}\text { D-Galactose-binding periplasmic } \\
\text { protein precursor }\end{array}$ & DGAL_SALTY & $m g l B / S T M 2190$ & $\begin{array}{l}\text { (K)GEPGH PDAEA R(T) } \\
\text { (K)ESGVI QGDLI AK(H) } \\
\text { (R)GQNVP VVFFN K(E) } \\
\text { (K)ALAIN LVDPA AAGTV IEK(H) } \\
\text { (R)VPYVG VDKDN LSEFT QK(E) }\end{array}$ & Fig. 3(a), spot 5 . \\
\hline $\begin{array}{l}\text { Dihydrolipoamide } \\
\text { dehydrogenase }\end{array}$ & Q8Z9E8 & lpdA/STM0154 & $\begin{array}{l}\text { (R)YDAVL VAIGR(V) } \\
\text { (R)VWDST DALEL K(E) } \\
\text { (K)GISYE TATFP WAASG R(A) } \\
\text { (K)VIPSI AYTEP EVAWV GLTEK(E) }\end{array}$ & Fig. 2(a) \\
\hline DnaK suppressor protein & DKSA_SALTY & $d k s A / S T M 0186$ & $\begin{array}{l}\text { (R)AAQEE EFSLE LR(N) } \\
\text { (R)NQLRDEVD(R) }\end{array}$ & Fig. 4(a) \\
\hline Probable thiol peroxidase & TPX_SALTY & tpx/STM1682 & (K)DLSDV SLSQY AGK(V) & \\
\hline Elongation factor Ts & EFTS_SALTY & tsf/STM0217 & $\begin{array}{l}\text { (K)AGNVA ADGVI K(T) } \\
\text { (K)DAGFQ AFADK }(\mathrm{V}) \\
\text { (K)KAGNV AADGV IK(T) } \\
\text { (K)DAGFQ AFADK VLDAA VAGK(I) }\end{array}$ & Fig. 3(a) \\
\hline $\begin{array}{l}\text { Fructose bisphosphate aldolase } \\
\text { class II }\end{array}$ & Q8ZL21 & STM3780 & $\begin{array}{l}\text { (K)IFDFV KPGVI TGDDV QK(V) } \\
\text { (-)SKIFD FVKPG VITGD DVQK(V) }\end{array}$ & Data not shown \\
\hline Hypothetical protein YciF & Q7CQF9 & yciF/STM1729 & $\begin{array}{l}\text { (R)DAALI AAAQK(V) } \\
\text { (K)TVEDL FIHLL SDTYS AEK(Q) } \\
\text { (K)VEHYE IASYG TLATL AEQLG } \\
\text { YSK(A) }\end{array}$ & Data not shown \\
\hline $\begin{array}{l}\text { Lysine, arginine, ornithine } \\
\text { (LAO)-binding periplasmic } \\
\text { protein }\end{array}$ & ARGT_SALTY & $\operatorname{argT/STM} 2355$ & $\begin{array}{l}\text { (K)EYAFA GPSVK(D) } \\
\text { (K)YFGDG TGVGL R(K) } \\
\text { (K)GSPIQ PTLES LK(G) } \\
\text { (K)GSPIQ PTLES LKGK(H) } \\
\text { (K)IADII SSLSI TDKR(Q) } \\
\text { (K)CTWVA SDFDA LIPSL K(A) } \\
\text { (R)LDAAL QDEVA ASEGF LKQPA } \\
\text { GK(E) }\end{array}$ & Data not shown \\
\hline
\end{tabular}


Table 1. cont.

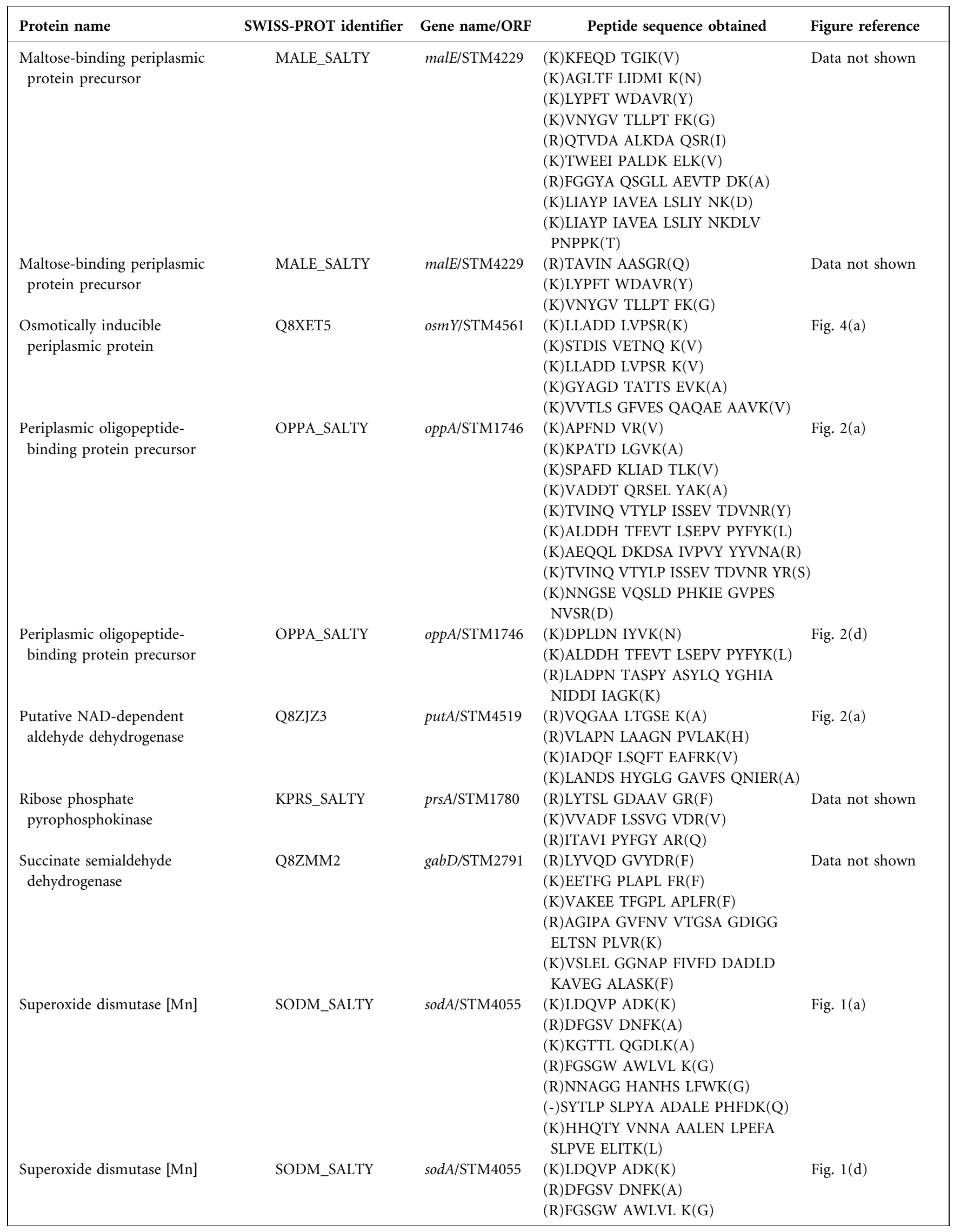


Table 1. cont.

\begin{tabular}{|c|c|c|c|c|}
\hline Protein name & SWISS-PROT identifier & Gene name/ORF & Peptide sequence obtained & Figure reference \\
\hline Transaldolase B & TALB_SALTY & talB/STM0007 & $\begin{array}{l}\text { (R)NNAGG HANHS LFWK(G) } \\
\text { (K)HHQTY VNNA AALEN LPEFA } \\
\text { SLPVE ELITK(L) } \\
\text { (R)ISTEV DAR(L) } \\
\text { (R)AQQVV DATDK(L) } \\
\text { (R)LSYDT EASIA K(A) }\end{array}$ & Fig. 3(a) \\
\hline
\end{tabular}

is more likely to find in its natural habitat, MglB acts as a glucose-scavenging system that can at least partially supplant PTS-dependent transport. This implies that MglB-dependent transport plays an important role in providing essential energy sources (glucose and galactose) for the survival of $S$. enterica in its natural environment. The presence of two isoforms of the substrate-binding protein MglB observed in this study, and their differential expression amongst the serovars, have not, to our knowledge, been described previously. Further studies could reveal if the two isoforms exhibit differential affinity to glucose or galactose, and how this contributes to the different phenotypes of the serovars of $S$. enterica.

The 2D GE profiles of the other serovars revealed that serovar Pullorum overexpressed the same isoform of $\mathrm{MglB}$ as Enteritidis but at a significantly lower level $(P<0.01)$, while in serovar Choleraesuis neither isoform was expressed at a detectable level (Fig. 3). Serovars Pullorum and Enteritidis are known to be evolutionarily closely related and are both common in infections associated with poultry eggs (Stanley \& Baquar, 1994). A mutation conferring an advantage for their survival, occurring after the divergence of these two serovars from the other groups, could have resulted in the differential expression of the two isoforms of $\mathrm{MglB}$. The absence of both isoforms from serovar Choleraesuis could be a result of low expression levels or post-translational modifications of $\mathrm{MglB}$ in this serovar. The gene for the transporter is present in the genome of serovar Choleraesuis, and shows $99 \%$ sequence homology when aligned with its homologue in serovar Typhimurium.

\section{Serovar Choleraesuis}

Comparison of the expression maps of serovars Typhimurium and Choleraesuis revealed one differentially expressed enzyme, succinate semialdehyde dehydrogenase I (GabD), encoded by gabD. This enzyme is a member of the aldehyde dehydrogenase family, comprising enzymes with aldehyde substrates, using NADP or NAD as a cofactor. Comparative analysis of the expression maps of serovar Typhimurium and Choleraesuis revealed that the enzyme was present in the Choleraesuis isolates but absent in the Typhimurium strains. None of the other serovars expressed

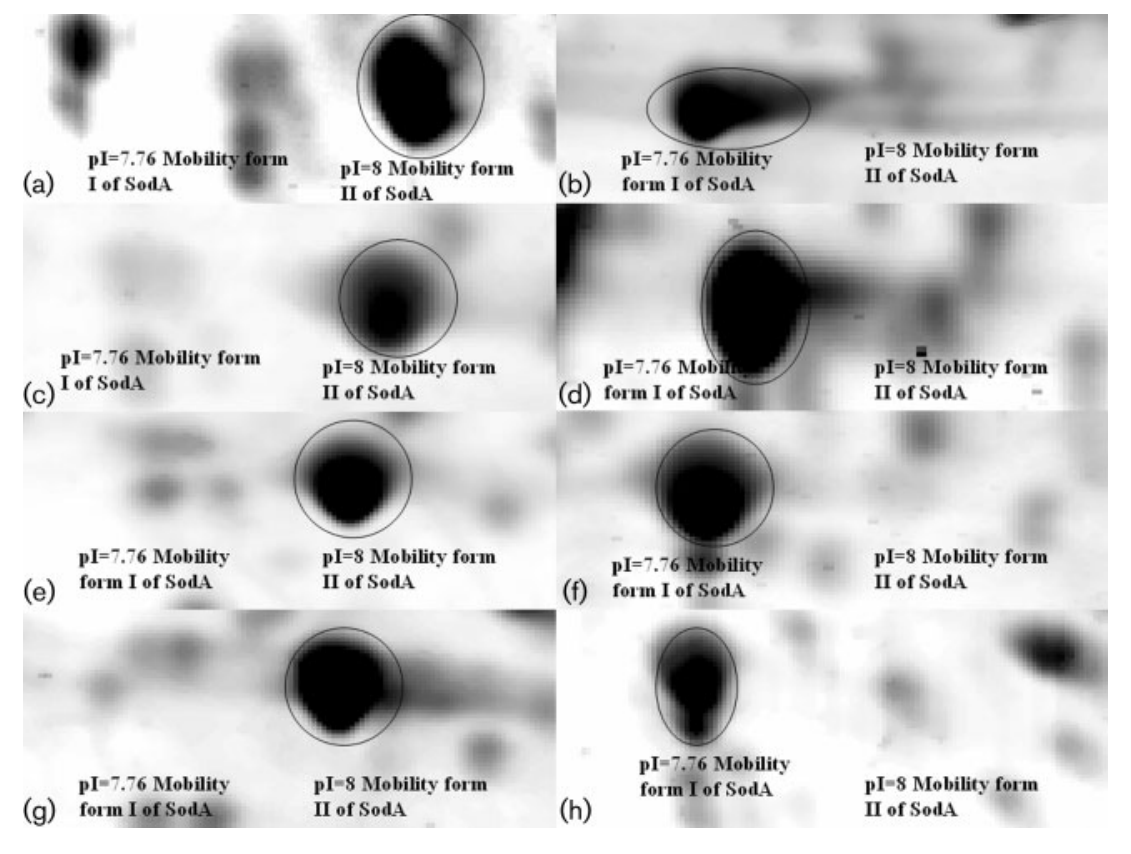

Fig. 1. Differential expression of the two isoforms of the $\mathrm{Mn}^{2+}$-binding superoxide dismutase (SodA) between serovar Typhimurium and the other Salmonella serovars. All four strains of serovar Typhimurium tested, 74 (a), 12023 (c), 204 (e) and 227 (g), overexpressed isoform II, while the strains representing the other serovars, serovar Pullorum B52 (b), serovar Choleraesuis B7 (d), serovar Enteritidis 12694 (f) and serovar Dublin 12709 (h) overexpressed isoform I. 


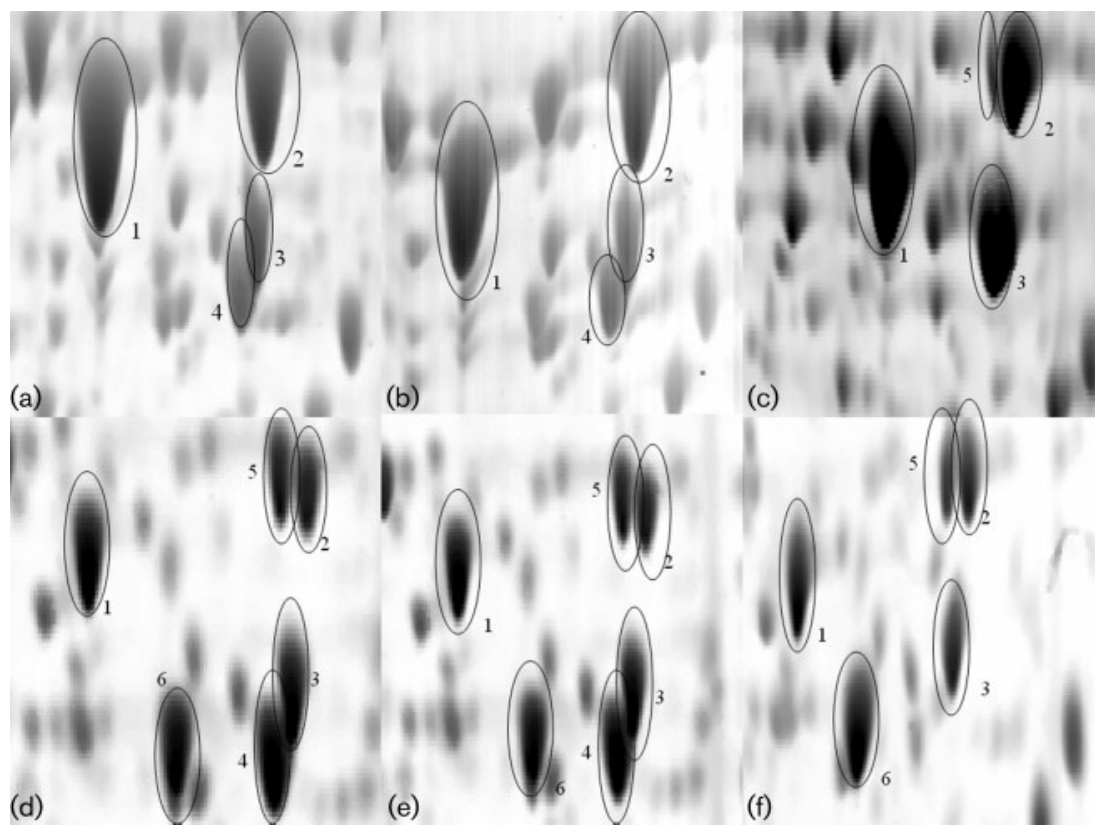

Fig. 2. Differential protein expression between reference strains and clinical isolates representing serovar Typhimurium including strains 74 (a and b), 12023 (c), 204 (d and e) and 227 (f). The reference strains 74 and 12023 showed high levels of expression of the two periplasmic transporters: ABC superfamily dipeptide transporter (1) and oligopeptidebinding protein (2) while the same proteins were expressed at significantly lower levels in the clinical isolates 204 and 227. The two enzymes dihydrolipoamide dehydrogenase (3) and putative NAD-dependent aldehyde dehydrogenase (4) did not show significant alteration in expression levels but the latter was not detected in the expression maps of 12023 and 227. Both clinical isolates, 204 and 227, showed increased levels of expression of two unknown proteins (5) and (6).

this enzyme, despite the fact that $g a b D$ is present in the genome of serovar Typhimurium. However, it is possible that the enzyme is modified in these serovars, and therefore possesses a different $\mathrm{pI}$ and molecular mass. However, our results correspond to other proteomic investigations (Coldham \& Woodward, 2004), where detailed proteome profiling of serovar Typhimurium has been performed using 2D LC-MS/MS, but GabD has not been detected. The products of the gabDTP locus ensure the transport and utilization of $\gamma$-aminobutyrate as a carbon and nitrogen source through the GABA pathway (Neidhardt, 1996) but the regulation of the operon has not been clearly defined. As all isolates have been subjected to identical growth conditions, the differences observed in this study indicate that the GabD enzyme is differentially regulated in serovar Choleraesuis.

\section{Serovar Pullorum}

Comparison of the profiles of serovar Typhimurium and Pullorum revealed differential expression of one of the isoforms of the lysine arginine ornithine (LAO)-binding transport protein. Two isoforms of this protein were detected in all strains of Typhimurium which were also present in all Pullorum isolates, but there was an additional isoform of LAO detected in all Pullorum isolates (data not shown). The three isoforms have identical molecular mass but differ greatly in $\mathrm{pI}$, by more than one $\mathrm{pH}$ unit. The LAO protein is the substrate-binding component of the Larginine uptake system in S. enterica, which functions through interaction with the HisP membrane complex (Neidhardt, 1996). Although this system is not well characterized, it is known that its expression is under nitrogen regulation (Kustu et al., 1979). As with the other isoforms observed for many of the transport proteins in this study, there is a strong indication of post-translational modification. The presence of several forms of the same transport protein may improve their affinity to the substrate. The presence of a third isoform of LAO specific for serovar Pullorum may confer an advantage on this serovar in its natural environment.

\section{Serovar Dublin}

The comparative analysis performed in this study did not reveal any proteins characteristic of this serovar. However, higher-resolution separation methods such as 'zoom-in' 2D gel gradients could help identify specific factors that were not observed in the current investigation.

\section{Expression differences related to source of the strains}

The comparative analysis of the profiles of all isolates tested revealed numerous differences in the expression levels of various proteins with unknown identity which could not be related to sero-specificity or host-adaptation. However, in some cases the differential expression levels appeared to correlate with the source of the isolates. Maltose-binding protein precursor (MalE) is a transport protein which was detected in two different mobility forms. Variation in the expression of only one of the isoforms was observed between strains. MalE was the second most prominent spot in the profile of the type strain of serovar Enteritidis, and was also one of the most prominent spots in the profiles of the type strains of serovars Typhimurium and Dublin. However, the clinical isolates of the same serovars, showed significantly lower levels of expression of this protein $(P<0.01)$. 

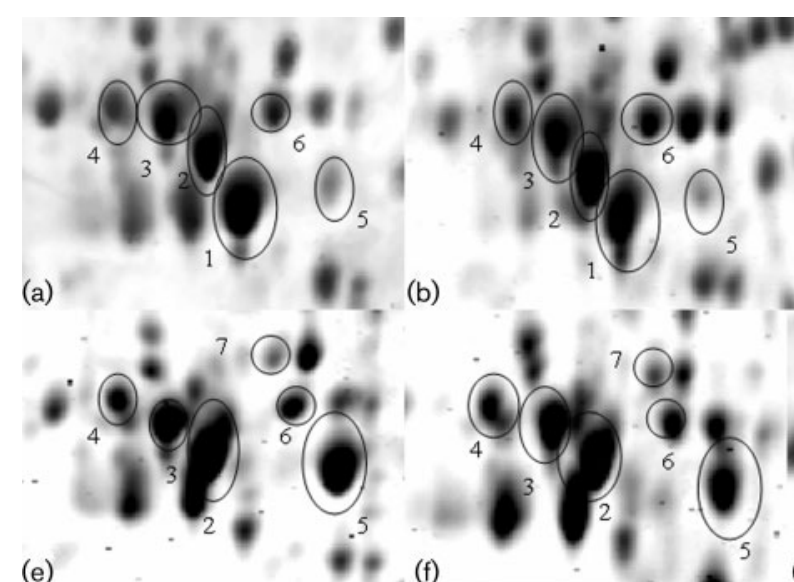

(b)
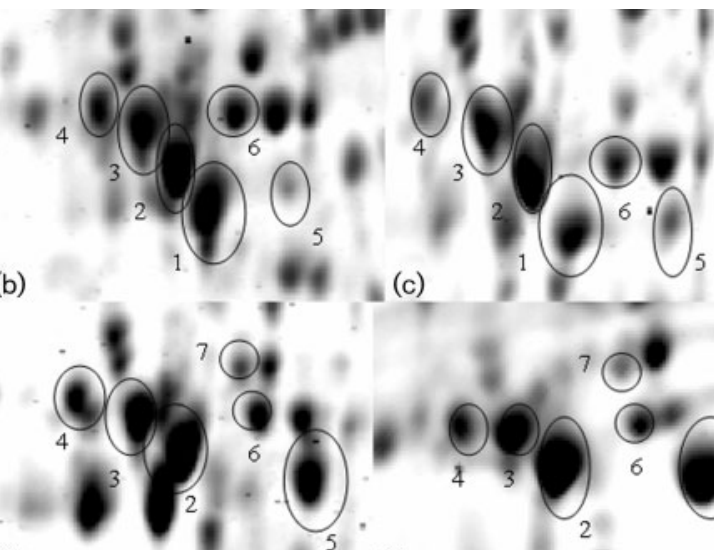

(c)
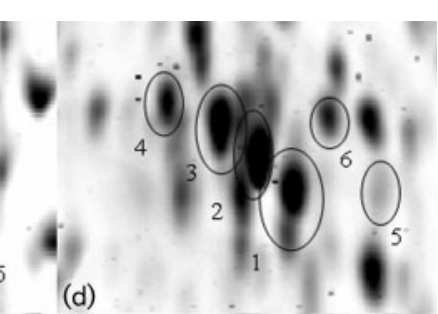

(d)

(f)
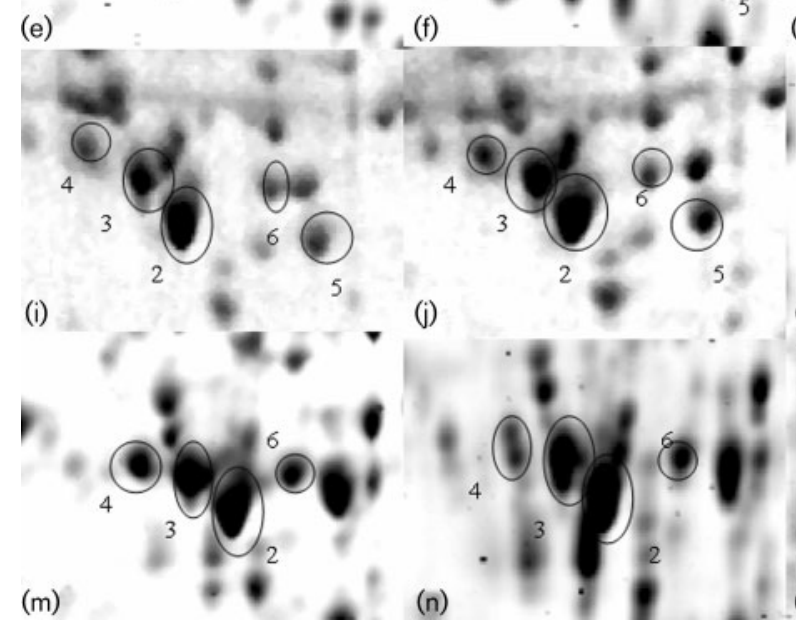

(k)

(g)
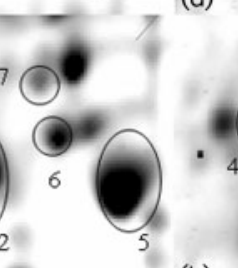

(h)
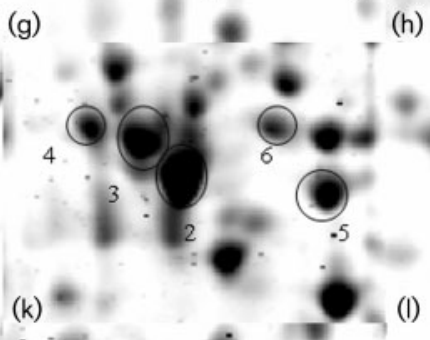

(I)

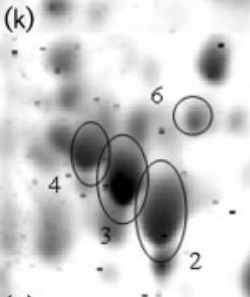

(o)

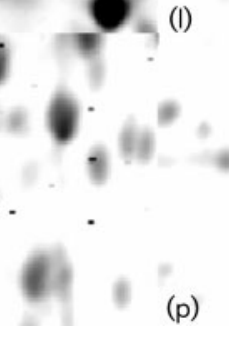

(p)
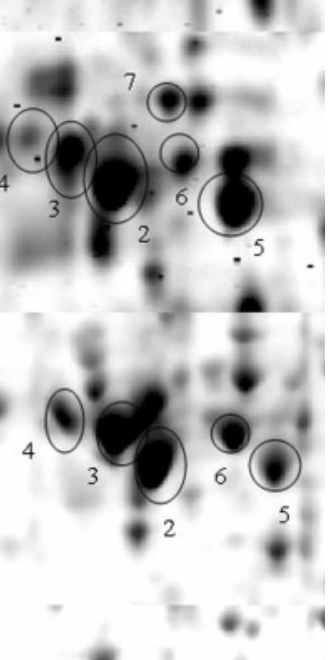

4

Fig. 3. Differential expression of $\mathrm{D}$-galactose-binding periplasmic protein precursor (MglB) isoforms amongst isolates representing serovars Typhimurium 74 ( $a$ and b) and 207 (c and d), serovar Enteritidis 12694 (e and f) and 97 ( $g$ and h), serovar Pullorum 10704 ( $i$ and j) and B52 ( $k$ and $)$ ), and serovar Choleraesuis B7 ( $m$ and $n$ ) and B6 (o and p). The first isoform (1) can be seen as a high-intensity spot in the expression maps of serovar Typhimurium while the second isoform (5) is present as a very faint spot. All isolates of serovar Enteritidis overexpress the second isoform (5). Serovar Pullorum also overexpressed the second isoforms while Choleraesuis expressed neither. The additional spots identified and labelled include (2) elongation factor Ts, (3) transaldolase B, (4) ADP-L-glycero-D-manno-heptose-6-epimerase, (6) ribose phosphate pyrophosphokinase.

MalE is an ATP-binding cassette (ABC) type transporter, which is part of the maltose/maltodextrin transport system found in Gram-negative bacteria (Boos \& Shuman, 1998). The malEFG operon is dependent on both the MalT regulatory protein and the cAMP/CAP complex, and is generally induced by the presence of its substrate or by free internal glucose. The complex regulation of this transport system is not fully understood and the presence of two differentially expressed isoforms of MalE, observed in this study, was not reported in a previous investigation (Boos \& Shuman, 1998). The high expression level of this protein in the laboratory-maintained isolates is indicative of a regulational change that is advantageous under those conditions.

Another protein with significantly higher levels of expression $(P<0.01)$ in the clinical isolates compared to reference strains was the cytosolic YciF protein, of unknown function (Fig. 4). YciF is a hypothetical protein, encoded by a gene that is part of the $y c i G F E-k a t N$ operon, whose expression has been reported to increase significantly when stimulated by bile (Prouty et al., 2004). The exact function of this protein and its role in S. enterica virulence is yet to be elucidated. It can be speculated that the elevated expression of this protein in the presence of bile (a factor Salmonella is likely to encounter when colonizing a host) indicates involvement in the process of invasion and survival in the host. This observation is also substantiated by the elevated expression of this protein in clinical isolates relative to the laboratory-maintained isolates.

\section{Conclusions}

Micro-organisms vary in their mechanisms of survival, some remaining at a particular site where nutrients are more favourable, while others are metabolically more versatile and disseminate more readily and consequently 


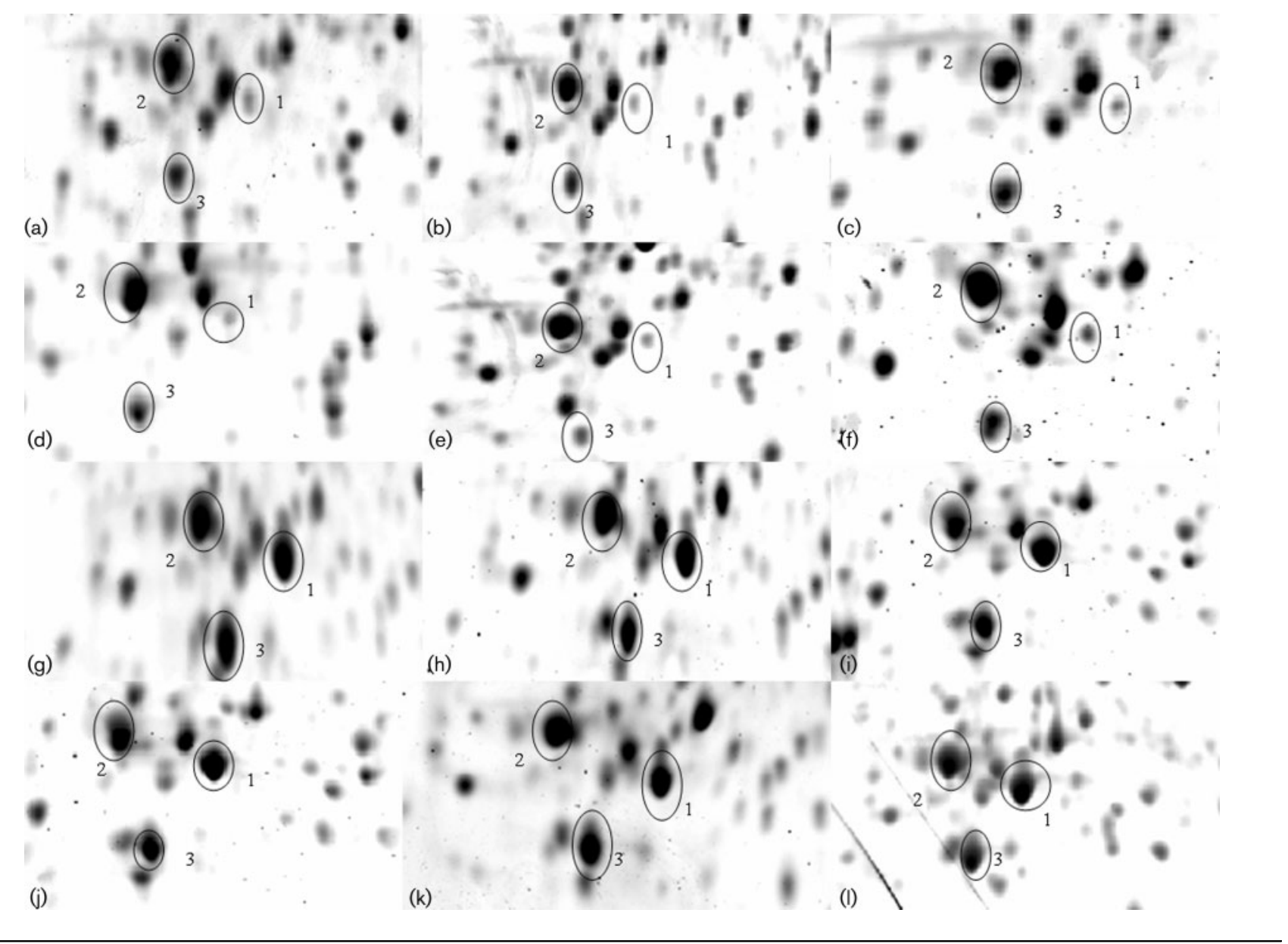

Fig. 4. Comparison of the expression of several low molecular mass proteins in the profiles of serovars Typhimurium (a, $d, g$ and j), Enteritidis (b, e, h and $\mathrm{k}$ ) and Dublin (c, f, i and l). The reference strains of these serovars are displayed on the top two rows and the clinical isolates on the bottom two rows. The DnaK suppressor protein DksA (3) and one of the forms of the putative periplasmic protein OsmY (2) were expressed by all strains with no significant change. However, all clinical isolates tested from all five serovars showed increased levels of expression of the hypothetical protein YciF (1), relative to the culture collection strains.

manifest different disease symptoms. Such differences in the capacity to spread and adapt to different conditions can be observed amongst the serovars of Salmonella enterica subspecies enterica (Kingsley et al., 2000; Kingsley \& Baümler, 2002). The existence of host-generalist and host-adapted variants of this species provides a unique opportunity to study the mechanisms defining the process of host adaptation, not only at the level of DNA sequences, but also at the level of the expressed proteome, which could reveal modifications and expression-level differences not detectable from the DNA data.

The comparison of the protein maps of the five serovars of $S$. enterica revealed high degrees of similarity that can be expected from isolates belonging to the same species. The majority of the observed proteins identified previously in the reference map of $S$. enterica serovar Typhimurium (Encheva et al., 2005) were expressed by all serovars, with no significant variation. Such proteins included glycolitic and Krebs cycle enzymes, chaperones, elongation factors and ribosomal proteins. Such proteins appear to be the 'core', or housekeeping proteome of this species, and are essential for its metabolism, transcription, protein synthesis and replication. While these proteins were expressed by both clinical and reference strains of all serovars with no change in expression levels, other proteins exhibited significant variation amongst different serovars and, in some cases, amongst isolates of the same serovar. This was particularly true for the transport proteins observed in the 2D GE profiles. Most of the transport proteins identified in this study were present in more than one isoform. These include OppA, which is involved in the transport of short peptides and was present in four isoforms, DppA, which is involved in the transport of dipeptides and was present in two isoforms, MalE, which is involved in the transport of maltose and was present in two isoforms, and $\mathrm{MglB}$, 
transporting galactose and glucose, also present in two isoforms. MglB showed a very distinct expression pattern, which was different in all serovars but had a high degree of similarity between Enteritidis and Pullorum.

The presence of several isoforms of the same protein is a common characteristic of $2 \mathrm{D}$ expression maps of bacteria. The majority of the published reference maps of various species demonstrate the presence of multiple spots identified as variants of the same gene product (Encheva et al., 2005; Cordwell et al., 2002; Voigt et al., 2004). This is usually assumed to be indicative of post-translational modifications but in this study some of the isoforms appeared only in a particular serovar or strain (e.g. SodA) and could also be the result of amino acid substitutions. This suggests that protein isoforms resulting from either post-translational modifications or amino acid substitutions are common in bacteria and appear to contribute greatly to the overall level of protein diversity observed amongst the isolates of $S$. enterica.

Some of the transport proteins studied here showed significant expression changes in only one of the isoforms, indicating a change in the ratio at which the isoforms are present. Overall, it appeared that the transport proteins were significantly overexpressed in the reference isolates compared to the clinical isolates. This was particularly true in the reference isolates obtained from the NCTC, which showed several-fold increases in expression levels of OppA, DppA and MalE. This finding suggests that differences observed cannot always be related to the serology, host specificity or evolution, but may also be related to the origin of the isolates and the effect of various external factors such as media composition, storage and temperature. The continuous subculturing that strains kept in culture collections undergo may favour mutations leading to regulation changes that would otherwise not be advantageous in the natural environment. However, despite the variation between isolates of different origin, several proteins that appeared to be serovar-specific were also observed. Despite the difficulty in ascertaining the reasons why these proteins are expressed by one serovar but not another, and how their function is related to hostspecificity, their identification demonstrates the ability of protein-based analysis to reveal specific biomarkers that it would not be possible to predict using the genomic data alone. The expression differences described here could also serve as potential diagnostic targets, but further validation of their serovar-specific expression on larger number of isolates is required. These findings provide the basis for further, more detailed, analysis of the serovar-specific differences reported in this study and the potential roles of these proteins in host-adaptation and pathogenicity.

\section{REFERENCES}

Baümler, A. J. (1997). The record of horizontal gene transfer in Salmonella. Trends Microbiol 5, 318-322.
Baümler, A. J., Tsolis, R. M., Fight, T. A. \& Adams, L. G. (1998). Evolution and host adaptation of Salmonella enterica. Infect Immun 66, 4579-4587.

Boos, W. \& Shuman, H. (1998). Maltose/maltodextrin system of Escherichia coli: transport, metabolism, and regulation. Microbiol Mol Biol Rev 62, 204-229.

Coldham, N. G. \& Woodward, M. J. (2004). Characterisation of Salmonella typhimurium proteome by semi-automated two-dimensional HPLC-mass spectrometry: detection of proteins implicated in multiple antibiotic resistance. J Proteome Res 3, 595-603.

Cordwell, S. J., Larsen, M. R., Cole, R. T. \& Walsh, B. J. (2002). Comparative proteomics of Staphylococcus aureus and the response of methicillin-resistant and methicillin-sensitive strains to Triton X-100. Microbiology 148, 2765-2781.

Crosa, J. H., Brenner, D. J., Ewing, W. H. \& Falkow, S. (1973). Molecular relationships among Salmonelleae. J Bacteriol 115, 307-315.

Death, A. \& Ferenci, T. (1993). The importance of the bindingprotein-dependent $\mathrm{Mgl}$ system to the transport of glucose in Escherichia coli growing on low sugar concentrations. Res Microbiol 144, 529-537.

Edwards, R. A., Olsen, G. J. \& Maloy, S. R. (2002). Comparative genomics of closely related salmonellae. Trends Microbiol 10, 94-100.

Encheva, V., Gharbia, S. E., Wait, R., Begum, S. \& Shah, H. N. (2005). Proteome analysis of serovars Typhimurium and Pullorum of Salmonella enterica subspecies I using two dimensional gel electrophoresis and LC/MS/MS. BMC Microbiol 5, 42.

Jeno, P., Mini, T., Moes, S., Hintermann, E. \& Horst, M. (1995). Internal sequences from proteins digested in polyacrylamide gels. Anal Biochem 224, 75-82.

Kingsley, R. A. \& Baümler, A. J. (2002). Pathogenicity islands and host adaptation of Salmonella serovars. Curr Top Microbiol Immunol 264, 67-87.

Kingsley, R. A., van Amsterdam, K., Kramer, N. \& Baümler, A. J. (2000). The shdA gene is restricted to serotypes of Salmonella enterica subspecies I and contributes to efficient and prolonged fecal shedding. Infect Immun 68, 2720-2727.

Kustu, S. G., McFarlend, N. C., Hui, S. P., Esmon, B. \& Ames, G. F.-L. (1979). Nitrogen control of Salmonella typhimurium: co-regulation of synthesis of glutamine synthetase and amino acid transport systems. J Bacteriol 138, 218-234.

Neidhardt, F. C. (1996). Escherichia coli and Salmonella: Cellular and Molecular Biology, vol. 1. Washington, DC: American Society for Microbiology.

O'Connor, C. D., Farris, M., Fowler, R. \& Qi, S. Y. (1997). The proteome of Salmonella enterica serovar Typhimurium: current progress on its determination and some applications. Electrophoresis 18, 1483-1490.

Park, M. R., Lee, E. G., Kim, Y. H., Jung, T. S., Shin, Y. S., Shin, G. W., Cha, H. G. \& Kim, G. S. (2003). Reference map of soluble proteins from Salmonella enterica serovar Enteritidis by two-dimensional electrophoresis. J Vet Sci 4, 143-149.

Perkins, D. N., Pappin, D. J. C., Creasy, D. M. \& Cottrell, J. S. (1999). Probability-based protein identification by searching sequence databases using mass spectrometry data. Electrophoresis 20, 3551-3567.

Popoff, M. Y., Bockemuhl, J. \& Gheesling, L. L. (2003). Supplement 2001 (no. 45) to the Kauffmann-White scheme. Res Microbiol 154, 173-174.

Prouty, A. M., Brodsky, I. E., Falkow, S. \& Gunn, J. S. (2004). Bile-salt mediated induction of antimicrobial and bile resistance of Salmonella typhimurium. Microbiology 150, 775-783. 
Qi, S.-Y., Moir, A. \& O'Connor, D. C. (1996). Proteome of Salmonella typhimurium SL1344: identification of novel abundant cell envelope proteins and assignment to a two-dimensional reference map. J Bacteriol 178, 5032-5038.

Rabilloud, T., Valette, C. \& Lawrence, J. J. (1994). Sample application by in-gel rehydration improves the resolution of two-dimensional electrophoresis with immobilized $\mathrm{pH}$ gradients in the first dimension. Electrophoresis 15, 1552-1558.

Shevchenko, A., Wilm, M., Vorm, O. \& Mann, M. (1996). Mass spectrometric sequencing of proteins from silver-stained polyacrylamide gels. Anal Chem 68, 850-858.

Stanley, J. \& Baquar, N. (1994). Phylogenetics of Salmonella enteritidis. Int J Food Microbiol 21, 79-87.

Tsolis, R. M., Baümler, A. J. \& Heffron, F. (1995). Role of Salmonella typhimurium Mn-superoxide dismutase (SodA) in protection against early killing by J774 macrophages. Infect Immun 63, 1739-1744.

van der Straaten, T., Zulianello, L., van Diepen, A., Granger, D. L., Janssen, R. \& van Dissel, J. T. (2004). Salmonella enterica serovar
Typhimurium RamA, intracellular oxidative stress response, and bacterial virulence. Infect Immun 72, 996-1003.

Voigt, B., Schweder, T., Becher, D., Ehrenreich, A., Gottschalk, G., Feesche, J., Maurer, K.-H. \& Hecker, M. (2004). A proteomic view of cell physiology of Bacillus licheniformis. Proteomics 4, 1465-1490.

Wait, R., Gianazza, E., Eberini, I., Sironi, L., Dunn, M., Gemeiner, M. \& Miller, I. (2001). Proteins of rat serum, urine, and cerebrospinal fluid: VI. Further protein identifications and interstrain comparison. Electrophoresis 22, 3043-3052.

Wait, R., Miller, I., Eberini, I., Cairoli, F., Veronesi, C., Battocchio, M., Gemeiner, M. \& Gianazza, E. (2002). Strategies for proteomics with incompletely characterized genomes: The proteome of Bos taurus serum. Electrophoresis 23, 3418-3427.

Wilm, M., Shevchenko, A., Houthaeve, T., Breit, S., Schweigerer, L. \& Fotsis, T. (1996). Femtomole sequencing of proteins from polyacrylamide gels by nano-electrospray mass spectrometry. Nature $\mathbf{3 7 9}$, $466-469$.

Edited by: P. H. Everest 